\title{
Verleihung des Förderpreises des SETAC-GLB an junge Nachwuchswissenschaftler/innen 2009
}

\author{
Eric Bruns • Anja Coors • Klaus Peter Ebke • Bettina Hitzfeld • Henner Hollert • Udo Hommen • Werner Manz • \\ Tobias Frische $\cdot$ Martina Ross-Nickoll
}

Eingegangen: 22. September 2010/ Akzeptiert: 22. September 2010/Online veröffentlicht: 21. Oktober 2010

(C) Springer-Verlag 2010

Der SETAC-GLB fördert seit mehreren Jahren den wissenschaftlichen Nachwuchs durch die Verleihung von Förderpreisen. Die Preise werden jeweils im Rahmen der Jahrestagungen überreicht. Die beste eingereichte Doktorarbeit wird hierbei mit einem Förderpreis in Höhe von $3000 €$ und die beste Diplom- oder Masterarbeit mit einem Förderpreis in Höhe von $1000 €$ gewürdigt. Die Preisverleihung fand auf der Jahrestagung 2009 der SETAC-GLB in München-Wei-

E. Bruns $(\bowtie)$

Bayer CropScience Aktiengesellschaft, BCS AG-D-ETX 6620,

40789 Monheim, Deutschland

E-Mail: eric.bruns@bayercropscience.com

A. Coors

ECT Oekotoxikologie $\mathrm{GmbH}$,

Böttgerstrasse 2-14, 65439 Flörsheim/Main, Deutschland

K.P. Ebke

Institut für Gewässerschutz MESOCOSM GmbH,

Neu-Ulrichstein 5, 35315 Homberg (Ohm), Deutschland

B. Hitzfeld

Bundesamt für Umwelt, Abt. Abfall, Stoffe, Biotechnologie,

3003 Bern, Schweiz

H. Hollert · M. Roß-Nickoll

Institut für Umweltforschung (Biologie V),

Rheinisch-Westfälische Technische Hochschule Aachen,

Worringerweg 1, 52074 Aachen, Deutschland

U. Hommen

Fraunhofer Institut für Molekularbiologie und Angewandte Ökologie, Auf dem Aberg 1, 57377 Schmallenberg, Deutschland

W. Manz

Universität Koblenz-Landau,

Institut für Integrierte Naturwissenschaften,

Universitätsstraße 1, 56070 Koblenz, Deutschland

T. Frische

Umweltbundesamt Dessau, Fachgebiet IV 1.3 - Pflanzenschutzmittel,

Wörlitzer Platz 1, 06844 Dessau-Roßlau, Deutschland henstephan statt, auf der den Preisträgern auch die Möglichkeit geboten wurde, ihre Arbeiten einem größeren Publikum vorzustellen. Der Preis für die beste Doktorarbeit wurde vom Verband der Chemischen Industrie e. V. (http://www. vci.de) gestiftet.

Für die Nachwuchsförderpreise 2009 hatten sich insgesamt 15 Kandidatinnen und Kandidaten mit acht Doktorarbeiten und sieben Diplomarbeiten beworben. Die Auswahl der besten Arbeiten erfolgte durch die Mitglieder des Vorstandes des SETAC-GLB in einem zweistufigen Verfahren. Für die Auswahl der preiswürdigen Arbeiten waren die hohe wissenschaftliche Qualität sowie eine klare Darstellung der verwendeten Methoden und der erzielten Ergebnisse ausschlaggebend. Eine ansprechende und übersichtliche Strukturierung der Arbeit war ebenso wichtig wie eine aussagefähige Diskussion mit einer kritischen Bewertung der eigenen Ergebnisse. Es war auch in diesem Jahr wieder eine spannende Herausforderung für die Jury, die Preisträger aus den eingereichten Arbeiten auszuwählen. Das breite Themenspektrum aus allen Gebieten der Ökotoxikologie und Umweltchemie und die durchweg hohe Qualität der Bewerbungen machte die Entscheidung nicht leicht. Die Wahl fiel auf zwei Arbeiten aus dem umweltanalytischen Bereich und aus dem Themenfeld der Wirkungsforschung. Der Vorstand der SETAC-GLB gratuliert den Preisträgern 2009:

\section{Beste Doktorarbeit - Dr. Urs Schenker}

Dr. Urs Schenker von der ETH Zürich erhielt den Preis für die beste Dissertation mit dem Titel „The role of intermediate degradation products for the assessment of persistent organic pollutants in a global multi-media model“".

Urs Schenker wurde 1977 in Bern geboren und studierte Umweltwissenschaften und -ingenieurwesen an der Eidge- 
nössischen Technischen Hochschule in Lausanne (EPFL). Nach seiner Diplomarbeit, welche er mit einem Forschungsaufenthalt am MIT verband, arbeitete er zwei Jahre in der Privatwirtschaft. Im Jahre 2004 begann er seine Doktorarbeit an der Eidgenössischen Technischen Hochschule in Zürich (ETH Zürich) in der Forschungsgruppe von Prof. Hungerbühler unter Betreuung von PD Dr. Martin Scheringer. Im Rahmen seiner Dissertation forschte er auch drei Monate an der University of California in Berkeley. Seit 2009 arbeitet er am Nestlé Research Center in Lausanne im Bereich Ökobilanzierungen.

Im Rahmen seiner kumulativen Doktorarbeit (z.B. Schenker et al. 2007, 2008a,b, 2009) modellierte Urs Schenker das Abbauverhalten von Chemikalien in der Umwelt, insbesondere welche Bedeutung Zwischenprodukte für die Risikoabschätzung der Ursprungschemikalie darstellen. Da viele dieser Zwischenprodukte ähnliche Strukturen wie die Originalsubstanzen aufweisen, können sie auch ähnliche toxikologische Eigenschaften haben und in der Umwelt ähnlich persistent sein. Urs Schenker erweiterte in seiner Arbeit ein existierendes globales Umweltmodell (CliMoChem, Scheringer et al. 2000), um damit auch Zwischenprodukte berücksichtigen zu können. Existierende Risikoindikatoren für Persistenz, räumliche Ausbreitung und Verschmutzungspotenzial für die Arktis konnten mit dem neuen Modell erweitert und auch für Zwischenprodukte definiert werden. In einer Reihe von Fallstudien wurde das Modell anschließend getestet: Beim Abbau von Fluorotelomeralkoholen (FTOH) und Perfluorooktylsulfonamido-Ethanolen (FOSE) kann Perfluorooktanoat (PFO) entstehen, welches aber auch direkt emittiert wird. PFO wird in hohen Konzentrationen in der Arktis gemessen. Es stellt sich daher die Frage nach dem Ursprung der großen Mengen PFO in der Arktis. Einerseits könnte PFO direkt emittiert und ozeanisch in die Arktis transportiert werden, was ein sehr langsamer Prozess wäre. Andererseits könnten FTOH und FOSE (welche sehr viel flüchtiger als PFO sind) über die Atmosphäre in die Arktis transportiert und dort zu PFO abgebaut werden. Dieser Prozess wäre sehr viel schneller. In seiner Arbeit konnte Urs Schenker zeigen, dass direkte Emissionen von PFO etwa 100-mal stärker zu arktischen Kontamination beitragen als FTOH- und FOSE-Emissionen. Dies bedeutet, dass auch eine radikale Reduktion der PFO-Emissionen in den nächsten Jahren wahrscheinlich direkt nur geringe Auswirkungen auf die Konzentration von PFO in der Arktis haben wird, weil der ozeanische Transport von PFO in den nächsten Jahren noch weiterhin neues PFO in die Arktis bringen wird.

In einer weiteren Fallstudie wurde das Verhalten von polybromierten Diphenylethern (PBDEs) untersucht. Diese Substanzen werden als Flammhemmer in Textilien und Polymeren eingesetzt. Es wird allerdings vermutet, dass PBDEs Effekte auf das Hormonsystem haben. Niederbromierte PBDEs wurden daher in der Europäischen Union verboten, höherbromierte PBDEs sind aber noch immer zugelassen. Höherbromierte PBDEs werden jedoch unter Laborbedingungen in niederbromierte PBDEs abgebaut. Falls diese Prozesse auch in der Umwelt stattfänden, sollten vorsorglich auch höherbromierte PBDEs verboten werden. In seiner Doktorarbeit hat Urs Schenker das Verhalten von verschiedenen PBDEs modelliert. Dabei berücksichtigte er, dass höherbromierte PBDEs debromiert werden können. Die Modellergebnisse zeigen, dass höherbromierte PBDEs nur eine Nebenquelle für niederbromierte PBDEs sind, deren Konzentrationen in der Umwelt wird hauptsächlich durch direkte Emissionen bestimmt. Trotzdem werden die Konzentrationen von niederbromierten PBDEs nicht auf Null zurückgehen, sollten höherbromierte PBDEs als kontinuierliche Quelle für niederbromierte PBDEs weiterhin verwendet werden.

In einer dritten Fallstudie wurde das Verhalten von DDT und den Abbauprodukten DDE und DDD untersucht. Modellresultate wurden mit Umweltmessungen zur Prüfung des Modells verglichen. Unter der Annahme, dass DDT auch weiterhin zur Bekämpfung von Malaria verwendet wird, sagte das Modell dennoch eine starke Abnahme von der DDT-Konzentration in der Arktis vorher. In den Tropen hingegen wird die Entwicklung der DDT-Konzentration in der Umwelt stark davon abhängen, wieviel DDT für die Bekämpfung von Malaria weiterhin verwendet wird. Durch das Aufzeigen der räumlichen und zeitlichen Kopplung von Anwendung und Umweltbelastung wird die Bewertung von Chancen und Risiken der DDT-Anwendung bedeutend erleichtert.

\section{Beste Diplomarbeit - Dipl. Biol. Jens C. Otte}

Den Nachwuchspreis 2009 für die beste Diplomarbeit konnte Jens Otte für seine Arbeit „Cytochrom P450 in frühen Lebensstadien des Zebrabärblings" entgegennehmen, die er am Zoologischen Institut der Universität Heidelberg angefertigt hat.

Jens C. Otte, Jahrgang 1981, studierte an den Universitäten Heidelberg und Uppsala, Schweden. Im Jahr 2006 schloss er sein Studienjahr an der Universität Uppsala (Schwerpunkt Toxikologie/Ökotoxikologie) mit dem schwedischen Hochschulabschluss Filosophie Magister ab. Die entsprechende Abschlussarbeit fertigte er im Labor von Prof. Dr. Björn Brunström zu dem Thema „Induction of ethoxyrsorufin-O-deethylase (EROD) in the three-spined stickleback (Gasterosteus aculatus L.) exposed to extracts of sediments from the Danube River" an. Ein zweimonatiger Forschungsaufenthalt führte ihn im Jahr 2007 in die Arbeitsgruppe von Prof. Dr. Giesy und Prof. Dr. Markus Hecker, University of Saskatchewan, Kanada. Im Jahr 2008 folgte der Abschluss seines Biologiestudiums an der Universität 
Heidelberg mit der Diplomarbeit „Cytochrom P450 in frühen Lebensstadien des Zebrabärblings", die unter der Anleitung von Prof. Dr. Thomas Braunbeck und Prof. Dr. Henner Hollert angefertigt wurde. Seit Dezember 2008 arbeitet er im Rahmen seiner Promotion in der Arbeitsgruppe von Prof. Dr. Uwe Strähle am Institut für Toxikologie und Genetik des Karlsruher Instituts für Technologie.

Die Exposition von Fischen gegenüber polyzyklischen aromatischen Kohlenwasserstoffen (PAKs), Polychlordibenzo-p-dioxinen/furanen (PCDD/Fs) und Polychlorbiphenylen (PCBs) ist aufgrund ihres Potenzials, den Gesundheitsstatus von Fischen und auch von Fischpopulationen zusätzlich zur Mortalität in frühen Lebensstadien zu beeinflussen, von hoher ökologischer Relevanz. Die Toxizität von PAKs und planaren halogenierten aromatischen Kohlenwasserstoffen (pHAKs; PCDD/Fs und PCBs) beruht auf der Aktivierung des Arylhydrocarbon-Rezeptors (AhR) und der darauf folgenden Aktivierung der „AhR-Gen-Kaskade“ inklusive der Cytochrom P450 1-(CYP1)-Isoformen. Um die Rolle des AhR-Signalweges und der CYP1-Aktivität für die Toxizität von PAKs und pHAKs während der Embryonalentwicklung zu verstehen, ist es notwendig zu zeigen, dass die Komponenten dieses Stoffwechselweges exprimiert werden und aktiv sind. Das Ziel dieser Studie war daher die Entwicklung einer Methode, um CYP1-Aktivität in frühen Lebensstadien des Zebrabärblings nachzuweisen. Ebenfalls sollte das zeitlich-räumliche Muster basaler und induzierter CYP1-Aktivität während der Embryonalentwicklung des Zebrabärblings erfasst werden, um die Rolle des AhR-Signalweges bei der Vermittlung der Toxizität von pHAKs und PAKs besser zu verstehen. Zusätzlich sollte das zeitlich-räumliche Muster basaler und induzierter CYP1Aktivität mit existierenden Daten der endogenen Rolle des AhR und CYP1s verglichen werden, um deren Rolle in der Entwicklung des Zebrabärblings zu untersuchen.

In vorbereitenden Experimenten mit dem Fischeitest wurden toxische Effekte der AhR-Agonisten 2,3,7,8-Tetrachlordibenzo-p-dioxin und beta-Naphthoflavon bestimmt und optimale Testkonzentrationen für die folgenden Versuche ermittelt. Die weit verbreitete Bestimmung der 7-Ethoxyresorufin-O-deethylase-(EROD)-Aktivität als Nachweis für CYP1-Aktivität wurde unter Zuhilfenahme der konfokalen Mikroskopie und klassischer Spektrometrie weiterentwickelt und zum Nachweis in frühen Lebensstadien des Zebrabärblings angepasst.

Der gewählte Ansatz zur Weiterentwicklung der Untersuchung auf EROD-Aktivität führte zu einer einfach durchzuführenden und sensitiven Methode für den Nachweis des Biotransformationsenzyms auch in sehr frühen Lebensstadien. Basale und induzierte CYP1-Aktivität wurde zu allen untersuchten Zeitpunkten von 8 bis $128 \mathrm{~h}$ nach der Befruchtung nachgewiesen und trat während der Entwicklung des
Zebrabärblings in einem dynamisch zeitlich-räumlichen Muster auf. Der Nachweis erfolgte früher als bisherige Berichte zum Beginn der Expression von CYP1A mRNA in der Embryonalentwicklung des Zebrabärblings und einer als bislang verzögert beschriebenen Aktivität von CYP1A. So wurde CYP1-Aktivität bereits in allen Keimblättern während der Gastrulation nachgewiesen. Bei fortschreitender Differenzierung war sowohl induzierte als auch basale EROD-Aktivität in Anlagen und Geweben des cardiovaskulären Systems, im Exkretionstrakt, im Verdauungstrakt und in Teilen des Gehirns sowie in bestimmten Stadien auch im inneren Teil des Auges und der Ohrenanlage deutlich nachweisbar. Effekte im Fischeitest korrelierten mit dem zeitlich-räumlichen Auftreten und der Quantität der CYP1-Aktivität nach $32 \mathrm{~h}$ nach der Befruchtung und auch in späteren Stadien. Aus Tierschutzgesichtspunkten stellt die Messung von CYP1-Aktivität in den frühen Lebensstadien des Zebrabärblings einen möglichen Ersatz für die In-vivo-Messung von EROD mit adulten Fischen in ökotoxikologischen Tests dar. Der Nachweis basaler Aktivität von CYP1 in den frühen Lebensstadien des Zebrabärblings einschließlich der Gastrulation legt nahe, dass die Aktivität nicht nur mit der Detoxifikation von Fremdstoffen, sondern auch mit der normalen Entwicklung zusammenhängen kann. Mögliche Mechanismen von CYP1-Aktivität während der Gastrulation des Zebrabärblings werden in der Diplomarbeit diskutiert.

Ergebnisse der Diplomarbeit von Herrn Otte sind mittlerweile auch international veröffentlicht (Otte et al. 2010).

Über die SETAC-GLB Förderpreise 2010 berichten wir in der nächsten Ausgabe. Die Ausschreibung für die Preise 2011 erfolgt in Kürze auf den Internetseiten des GLB (http:// www.setac-glb.de).

\section{Literatur}

Otte J, Schmidt AD, Hollert H, Braunbeck T (2010) Spatio-temporal development of CYP1 activity in early life-stages stages of zebrafish (Danio rerio). Aquatic Toxicol 10:38-50

Schenker U, Scheringer M, Hungerbuehler K (2007) Including degradation products of persistent organic pollutants in a global multimedia box model. Environ Sci Poll Res 14:145-152

Schenker U, Soltermann F, Scheringer M, Hungerbuehler K (2008a) Modeling the environmental fate of polybrominated diphenyl ethers (PBDEs): The importance of photolysis for the formation of lighter PBDEs. Environ Sci Technol 42:9244-9249

Schenker U, Scheringer M, Hungerbühler K (2008b) Investigating the global fate of DDT: model evaluation and estimation of future trends. Environ Sci Technol 42:1178-1184

Schenker U, Scheringer M, Sohn MD, Maddalena RL, McKone TE, Hungerbuehler K (2009) Using information on uncertainty to improve environmental fate modeling: a case study on DDT. Environ Sci Technol 43:128-134

Scheringer M, Wegmann F, Fenner K, Hungerbühler K (2000) Investigation of the cold condensation of persistent organic chemicals with a global multimedia fate model. Environ Sci Technol 34:1842-1850 